\title{
Approach to MAI cancellation for micro-satellite clusters
}

\author{
HUANG Jiajun, ZHANG Chaojie*, and JIN Xiaojun \\ Micro-Satellite Research Center, Zhejiang University, Hangzhou 310027, China
}

\begin{abstract}
With the development of micro-satellite technology, traditional monolithic satellites can be replaced by micro-satellite clusters to achieve high flexibility and dynamic reconfiguration capability. For satellite clusters based on the frequency division-code division multiple access (FD-CDMA) communication system, the inter-satellite ranging precision is usually constrained due to the influence of multi-address interference (MAl). The multi-user detection (MUD) is a solution to MAI, which can be divided into two categories: the linear detector (LD) and the non-linear detector (NLD). The general idea of the LD is aiming to make a better decision during the symbol decision process by using the information of all channels. However, it is not beneficial for the signal phase tracking precision. Instead, the principle of the NLD is to rebuild the interference signal and cancel it from the original one, which can improve the ranging performance at the expense of considerable delays. In order to enable simultaneous ranging and communication and reduce multi-node ranging performance degradation, this paper proposes an NLD scheme based on a delay locked loop (DLL), which simplifies the receiver structure and introduces no delay in the decision process. This scheme utilizes the information obtained from the interference channel to reconstruct the interference signal and then cancels it from the original delayed signal. Therefore, the DLL input signal-to-interference ratio (SIR) of the desired channel can be significantly improved. The experimental results show that with the proposed scheme, the standard deviation of the tracking steady error is decreased from $5.59 \mathrm{~cm}$ to $3.97 \mathrm{~cm}$ for $\mathrm{SIR}=5 \mathrm{~dB}$, and $13.53 \mathrm{~cm}$ to $5.77 \mathrm{~cm}$ for $\mathrm{SIR}=-5 \mathrm{~dB}$, respectively.
\end{abstract}

Keywords: micro-satellite, satellite clusters, multi-user detection (MUD), interference cancellation, inter-satellite ranging.

DOI: $10.21629 /$ JSEE.2019.05.01

\section{Introduction}

Compared with traditional monolithic satellites [1], microsatellites [2] have the advantages of lower cost, rapid development and more flexibility for on-orbit task reconfiguration $[3,4]$. The satellite clusters $[5,6]$ consisting of multi-

\footnotetext{
Manuscript received December 19, 2018.

*Corresponding author.

This work was supported by the China National Funds of Distributed Young Scientists (61525403) and the Fundamental Research Funds for the Central Universities (2018QNA4053).
}

ple micro-satellites are becoming a better option over traditional satellites.

In general, the satellites in a cluster is relatively compact, which is beneficial for their mutual sensing ability. It is necessary to use a high-precision inter-satellite ranging system [7,8] and attitude-aware control system $[9,10]$ to avoid collisions between satellites and to maintain the spatial configuration. For satellite clusters based on the frequency division-code division multiple access (FDCDMA) [11] communication system, the inter-satellite ranging precision is often constrained due to the influence of multi-address interference (MAI) [12,13]. Meanwhile, the ranging result should be updated in time for the stability of the cluster configuration. To address these issues, multi-user detection (MUD) [14,15] techniques are often used in the satellite clusters based on the FD-CDMA communication system.

The existing and emerging MUD techniques can be divided into two categories, the linear detector (LD) and nonlinear detector (NLD). The LD is based on a traditional detector, and its output is linearly transformed to optimize the symbol decision. The widely used decorrelation detector $[16,17]$ only needs to know the inverse matrix of the cross-correlation matrix between channels, and no amplitude information is required. However, its noise is amplified while suppressing MAI. The minimum mean square error detector $[18,19]$ aims at minimizing the mean square error between the transmitted signal and its estimation. Its performance is better than the decorrelation detector in the same circumstance if the noise is taken into consideration, but the amplitude matrix must be known.

The NLD reconstructs the signal based on the information already obtained in each channel and cancels the interference signal from the original signal. The NLD attempts removal of MAI from each channel's received message before the decision process. It falls into two categories, namely, serial interference cancellation (SIC) [20,21] and parallel interference cancellation (PIC). Concerning the SIC, coordinated processing of the received signal with a 
successive cancellation scheme in which the interference caused by the remaining channels is removed from each channel in succession. As for PIC, it simultaneously removes from each channel the interference produced by the remaining channels. The ranging precision is determined by the pseudo-code phase tracking precision rather than the symbol decision performance. For the LD, a better decision is made at the symbol decision process, so the ranging precision cannot be improved. The NLD can improve the output SIR, so the pseudo-code tracking precision can be improved. However, existing NLD schemes inevitably introduce delays.

This paper proposes an NLD scheme based on delay locked loop (DLL), which simplifies the receiver structure and introduces no delay in the decision process. The scheme utilizes the estimation of the amplitude, pseudocode phase, carrier phase and symbol to reconstruct the interference. Compared to the LD, the output SIR of the desired channel can be significantly improved, not just the bit error rate (BER) after signal demodulation. Therefore, the ranging precision can be improved. More importantly, no additional delays will be introduced in the demodulation process compared to the NLD, so the real-time performance is guaranteed.

The remainder of this paper is organized as follows. The model and analysis of the scheme are discussed in Section 2. Simulation and experimental results are presented in Section 3 and Section 4, respectively. Finally, this paper is concluded in Section 5.

\section{System overview}

\subsection{Interference cancellation model}

Taking channel $k$ as the desired channel in the baseband part of a spread spectrum receiver, the proposed scheme is shown in Fig. 1.

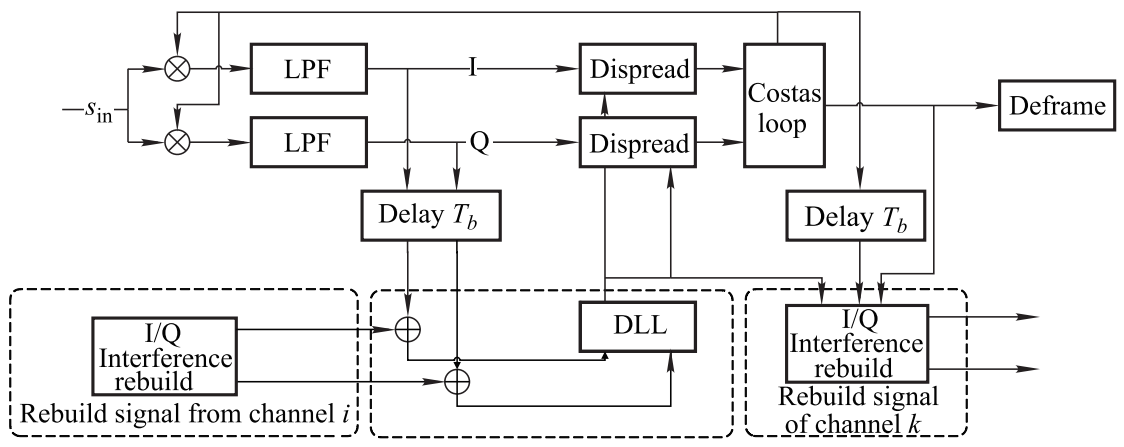

Fig. 1 Diagram of interference cancellation

The received signal $s_{\text {in }}(t)$ is divided into two paths after passing through the orthogonal down-convertor and low pass filter (LPF). One of the paths is dispreaded and then sent to the carrier recovery loop. In this system, a Costas loop is used as the carrier recovery loop, in which the carrier is tracked, and the symbol of channel $k$ is estimated.

After the signal of the other path is delayed by one symbol period time $T_{b}$, the interference component is cancelled by the reconstructed signal from channel $i$ to destruct the unwanted component from channel $k$. Then, the signal is sent to a DLL where the pseudo-code of the corresponding channel is tracked, and the prompt code is output for dispreading.

\subsection{Process of interference cancellation}

The proposed scheme is divided into two steps. Assuming that channel $k$ is the desired channel and channel $i$ is the interference channel, the first step is a process of reconstructing the signal of the previous $T_{b}$ of channel $i$. In the second step, the input signal of the DLL is delayed by one
$T_{b}$, after which the reconstructed signal in the first step is used to destruct the interference.

It is assumed that the symbols of each channel are aligned in time, and the received signals are not changing rapidly. Besides, the receiver performs the proposed scheme from the beginning of the first symbol period $T_{b 0}$ as Fig. 2 shows. In order to simplify the representation, only the symbol tracked by the carrier recovery loop $b_{k, n}(t)$ and the pseudo-code phase tracked by the DLL $c_{k, n}(t)$, where the subscript here indicates the $n$th symbol of channel $k$. The carrier recovery loop can only obtain the estimation of symbol value $\widehat{b}_{k, n}(t)$ at the beginning of next $T_{b}$. Since the received signal does not change rapidly, the local replica of the previous pseudo-code phase $\widehat{c}_{k, n}(t)$ can be used for the dispreading process of the current symbol. Therefore no delay is introduced in the demodulation process of the DLL. At the beginning, the signal $\widehat{b}_{k, 0}(t) \cdot \widehat{c}_{k, 0}(t)$ of channel $k$ during $T_{b 0}$ can be reconstructed, and it can be used to destruct the components of channel $k$. 


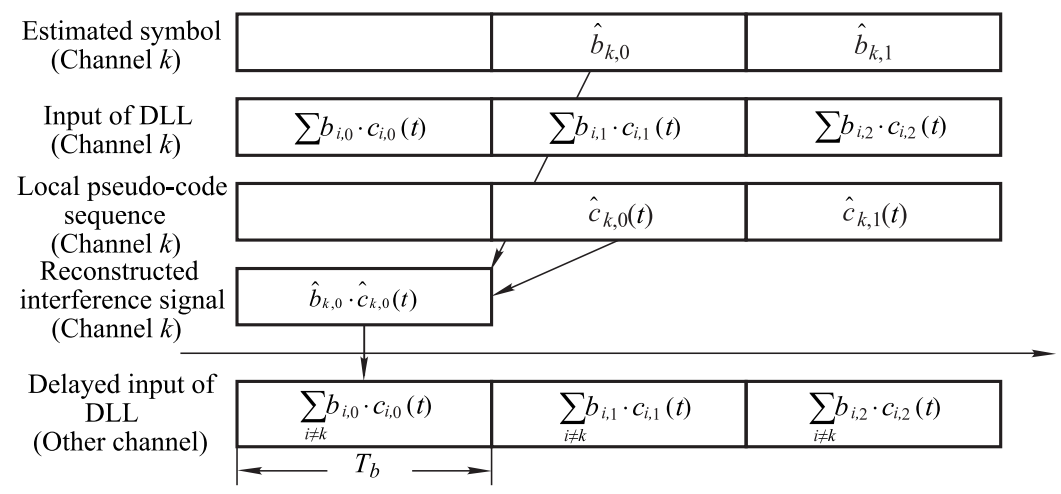

Fig. 2 Process of interference cancellation

Similarly, interference components from other channels can also be eliminated in channel $k$. In order to simplify the discussion, taking a two-channel case as an example. After the proposed scheme is performed in channel $k$, the result of the leading branch $I_{E}$ is

$$
I_{E}=\frac{1}{T_{b}} \int_{0}^{T_{b}} s_{k, n-1}(t) \widehat{c}_{k, n-2}\left(t-\tau_{E}\right) \mathrm{d} t+
$$

$\frac{1}{T_{b}} \int_{0}^{T_{b}}\left[s_{i, n-1}(t)-\widehat{s}_{i, n-1}(t)\right] \widehat{c}_{k, n-2}\left(t-\tau_{E}\right) \mathrm{d} t=\alpha_{k}+\varepsilon$

where

$$
\left\{\begin{array}{l}
s_{k, n-1}(t)=\frac{A_{k, n-1}}{2} b_{k, n-1}(t) c_{k, n-1}(t) \cos \varphi_{k, n-1}(t) \\
\varphi_{k, n-1}(t)=\theta_{k, n-1}(t)-\theta_{i, n-1}(t) \\
\alpha_{k}=\frac{1}{T_{b}} \int_{0}^{T_{b}} s_{k, n-1}(t) \widehat{c}_{k, n-2}\left(t-\tau_{E}\right) \mathrm{d} t \\
\varepsilon=\frac{1}{T_{b}} \int_{0}^{T_{b}}\left[s_{i, n-1}(t)-\widehat{s}_{i, n-1}(t)\right] \widehat{c}_{k, n-2}\left(t-\tau_{E}\right) \mathrm{d} t
\end{array}\right.
$$

where $A$ is the amplitude of the signal, $\tau_{E}$ is the leading correlation time, $\varepsilon$ is the error residual, and $\theta$ is the carrier phase.

\subsection{Analysis of main error residuals}

\subsubsection{Pseudo-code phase error residual}

It is assumed that other estimations are accurate and interference channel $i$ is already locked. The phase difference between $\widehat{c}_{i}(t)$ and $c_{i}(t)$ can be converted to a time difference written as

$$
\widehat{c}_{i}(t)=c_{i}(t+\Delta \tau)
$$

where $\Delta \tau$ is the time difference between $\widehat{c}_{i}(t)$ and $c_{i}(t)$.

Substituting (3) into (1) yields a cancellation error residual $\varepsilon_{C}$ caused by the pseudo-code phase estimation error

$$
\varepsilon_{C}=\frac{A_{i}}{2 T_{b}} b_{i, n-1} \cos \varphi_{i} .
$$

$$
\int_{0}^{T_{b}}\left[c_{i}(t)-c_{i}(t+\Delta \tau)\right] \widehat{c}_{k}\left(t-\tau_{E}\right) \mathrm{d} t .
$$

It can be inferred that the variance of the pseudo-code phase estimation error introduced by the DLL of channel $i$ can be written as

$$
D\left[\varepsilon_{c}\right]=\frac{A_{i}^{2}}{4} \cdot \frac{\sigma_{\mathrm{DLL} i}^{2}}{T_{c}^{2}}
$$

where $D[\cdot]$ indicates the variance of the contents in the square brackets, $\sigma_{\mathrm{DLL} i}$ is the standard variance of the DLL tracking error of channel $i$ and $T_{C}$ is the pseudo-code chip period time.

It is assumed that the pseudo-code sequences used in each channel are of the same family. Referring to [22], the relationship between $\varepsilon_{C}$ and the equivalent carrier-to-noise ratio (CNR) of channel $i$ can be expressed as

$$
D\left[\varepsilon_{c}\right]=\frac{A_{i}^{2}}{4 N} \cdot \sigma_{\mathrm{DLL} i}\left(C_{i} / N_{e q}\right)
$$

where $N$ is the length of the pseudo-code sequence and $C_{i} / N_{e q}$ is the receiver input CNR, $N_{e q}$ is the equivalent noise power, and $C_{i}$ is the desired signal power. According to [14], $\sigma_{\mathrm{DLL} i}$ can be written as

$$
\sigma_{\mathrm{DLL} i}=\sqrt{\frac{B_{L p}}{2 B_{f e} \cdot C_{i} / N_{e q}}\left(1+\frac{1}{T_{\mathrm{cohp}} \cdot C_{i} / N_{e q}}\right)}
$$

where $B_{L p}$ is the bandwidth of the equivalent thermal noise of the DLL, and $T_{\text {cohp }}$ is the coherent integration time of the DLL.

The output $\operatorname{SIR~SIR}_{C}$ can be expressed as

$$
\operatorname{SIR}_{C}=\frac{\mathrm{E}\left[I_{E}\right]^{2}}{D\left[\varepsilon_{C}\right]}=\frac{A_{k}^{2}}{A_{i}^{2}} \cdot \frac{N}{4 \sigma_{\mathrm{DLL} i}}
$$

where $\mathrm{E}[\cdot]$ indicates the mathematical expectation of the contents in the square brackets. 


\subsubsection{Carrier phase error residual}

The carrier phase error residual can be expressed as

$$
\varepsilon_{\varphi}=\frac{A_{i}}{2}\left(\cos \varphi_{i}-\cos \widehat{\varphi}_{i}\right)
$$

The interference signal can be considered as an equivalent thermal noise [23]. According to [24], the standard variance of the carrier tracking error $\sigma_{\mathrm{PLL}}$ can be expressed as

$$
\sigma_{\mathrm{PLL}}=\sqrt{\frac{B_{L}}{C / N_{e q}} \cdot\left(1+\frac{1}{2 T_{\mathrm{coh}} \cdot C / N_{e q}}\right)}
$$

where $B_{L}$ is the bandwidth of the equivalent thermal noise of the carrier recovery loop, and $T_{\text {coh }}$ is its coherent integration time.

If the standard variance of the equivalent thermal noise of channel $i$ and channel $k$ is $\sigma_{\mathrm{PLL} i}$ and $\sigma_{\mathrm{PLL} k}$, then the standard variance of the difference between them can be expressed as

$$
\sigma_{\mathrm{PLL}}=\sqrt{\sigma_{\mathrm{PLL} i}^{2}+\sigma_{\mathrm{PLL} k}^{2}}
$$

It can be assumed that the phase tracking error of the Costas loop obeys the normal distribution and $\varphi_{i}$ is uniformly distributed so that the variance $D\left[\varepsilon_{\varphi}\right]$ can be expressed as

$$
D\left[\varepsilon_{\varphi}\right]=\frac{A_{i}^{2}}{4} \cdot D\left[\cos \varphi_{i}-\cos \widehat{\varphi}_{i}\right]=\frac{2 A_{i}^{2}}{\pi^{2}} \cdot \sigma_{\mathrm{PLL}}^{2} .
$$

The output $\operatorname{SIR~SIR}_{\varphi}$ can be expressed as

$$
\operatorname{SIR}_{\varphi}=\frac{\mathrm{E}\left[I_{E}\right]^{2}}{D\left[\varepsilon_{\varphi}\right]}=\frac{A_{k}^{2}}{A_{i}^{2}} \cdot \frac{\pi^{2}}{32 \sigma_{\mathrm{PLL}}^{2}} .
$$

\subsubsection{Amplitude residual}

The error residual introduced by the amplitude estimation error can be expressed as

$$
\varepsilon_{A}=\left(\frac{\widehat{A}_{i}}{2}-\frac{A_{i}}{2}\right) b_{i, n-1} \cos \varphi_{i}
$$

Due to the tracking error of the DLL, the autocorrelation value of channel $i R_{i}(\Delta \tau)$ does not remain constant at the autocorrelation peak.

When a gold code with a high order is used as the pseudo-code sequence [16], $R_{i}(\Delta \tau)$ can be expressed as

$$
R_{i}(\Delta \tau)=1-\frac{|\Delta \tau|}{T_{C}}
$$

It can be assumed that $\Delta \tau$ obeys the normal distribution $N\left(0, \sigma_{\mathrm{PLL}}^{2}\right)$. According to [25], the mathematical expectation of $R_{i}(\Delta \tau)$ can be written as

$$
\begin{gathered}
\mathrm{E}\left[R_{i}(\Delta \tau)\right]=\int_{-\infty}^{+\infty}\left(1-\frac{|\Delta \tau|}{T_{c}}\right) \cdot f_{\mathrm{PDF}}(\Delta \tau) \mathrm{d} \Delta \tau= \\
1-\sqrt{\frac{2}{\pi} \cdot \sigma_{\mathrm{DLL} i}}
\end{gathered}
$$

where $f_{\mathrm{PDF}}$ is the probability density function of normal distribution.

Moreover, the amplitude estimation can be expressed as

$$
\widehat{A}_{i}=A_{i} \cdot\left[1-\sqrt{\frac{2}{\pi} \cdot \sigma_{\mathrm{DLL} i}}\right] .
$$

Therefore, the output $\mathrm{SIR} \mathrm{SIR}_{A}$ can be expressed as

$$
\operatorname{SIR}_{A}=\frac{\mathrm{E}\left[I_{E}\right]^{2}}{D\left[\varepsilon_{A}\right]}=\frac{A_{i}^{2}}{A_{i}^{2}} \cdot \frac{\pi N}{4 \sigma_{\mathrm{DLL} i}} .
$$

\subsection{Comparison of error residuals}

With the proposed scheme, the SIR of the desired channel $\mathrm{SIR}_{P}$ will increase compared to the original SIR SIR $\mathrm{Sin}_{\text {. }}$ The cancellation gain $G$ can be defined as

$$
G=\operatorname{SIR}_{P}-\operatorname{SIR}_{\text {in }} .
$$

In practice, since the signal bandwidth needs to include the main lobe and the primary side lobes on both sides of the spread spectrum modulation signal, the bandwidth of the analog frontend can be approximated as $4 f_{\mathrm{PN}}$, where $f_{\mathrm{PN}}$ is the pseudo-code chip rate. Thus $4 f_{\mathrm{PN}} N_{0}$ can be considered as the in-band noise power of the signal, where $N_{0}$ is the noise floor of the receiver. Furthermore, referring to the practical engineering experience of [26], the MAI power of the same pseudo-code sequence family can be equivalent to twice the power of the in-band white noise. Therefore, $C / N_{e q}$ can be expressed as

$$
C / N_{e q}=\frac{C \cdot B_{f e}}{B_{f e} N_{0}+2 J_{\Sigma}}
$$

where $J_{\Sigma}$ is the sum of the power of the MAI and $B_{f e}=$ $4 f_{\mathrm{PN}}$ is the bandwidth of the analog frontend.

In order to verify the effectiveness of the proposed scheme, experiments are conducted under strong signal power condition. In this situation, the influence of the receiver noise floor can be neglected, so the output SIR of various residuals can be simplified as follows:

$$
\left\{\begin{array}{l}
\operatorname{SIR}_{C}=\frac{N T_{C} B_{f e}^{2}}{4 B_{L}} \\
\operatorname{SIR}_{\varphi}=10 \lg \left(\frac{\pi^{2} B_{f e}}{64 B_{L p}}\right) . \\
\operatorname{SIR}_{A}=\frac{\pi N T_{C} B_{f e}^{2}}{4 B_{L p}}
\end{array}\right.
$$


Substituting the experimental parameters into (21) yields

$$
\left\{\begin{array}{l}
G_{\mathrm{SIR}_{C}}=83-\mathrm{SIR}_{\mathrm{in}} \\
G_{\mathrm{SIR}_{\varphi}}=28-\mathrm{SIR}_{\mathrm{in}} \\
G_{\mathrm{SIR}_{A}}=88-\mathrm{SIR}_{\mathrm{in}}
\end{array} .\right.
$$

It can be seen from (22) that the carrier phase residual has the most significant influence on the proposed scheme, which can be considered as the main limiting factor of the cancellation gain. Therefore, the carrier phase error residual can be used to estimate the theoretical ranging precision of the proposed scheme.

\section{Simulation results}

Simulink [27] is used to verify the effectiveness and performance of the proposed scheme. The Simulink version used in this simulation platform is Matlab 2012b. The primary parameters of the simulation platform are shown in Table 1.

Table 1 Primary parameters of simulation

\begin{tabular}{cc||cc}
\hline Parameter & Value & Parameters & Value \\
\hline$f_{s} / \mathrm{MHz}$ & 40 & $B_{L} / \mathrm{kHz}$ & 1 \\
$f_{c} / \mathrm{MHz}$ & 10 & $\xi_{L}$ & 0.7071 \\
$f_{\mathrm{PN}} / \mathrm{MHz}$ & 5.115 & $B_{L p} / \mathrm{Hz}$ & 200 \\
$f_{b} / \mathrm{kHz}$ & 5 & $\xi_{L p}$ & 0.7071 \\
$N$ & 1023 & & \\
$\mathrm{SNR} / \mathrm{dB}$ & 100 & & \\
\hline
\end{tabular}

$f_{s}$ is the system clock frequency, $f_{c}$ is the carrier frequency, $f_{b}$ is the symbol rate, $\xi_{L}$ is the damping factor of the carrier tracking loop, and $\xi_{L p}$ is the damping factor of DLL.

There are two independent transmitters Tx1 and Tx2, which use two different pseudo-code sequences of the same family. The output power of Tx 1 can be adjusted as needed, while the output power of $T \times 2$ remains fixed. The combined output signal of $\mathrm{Tx} 1$ and $\mathrm{Tx} 2$ is delivered to the receivers $\mathrm{Rx} 1$ and $\mathrm{Rx} 2$. The local pseudo-code sequences of $\mathrm{Rx} 1$ and $\mathrm{Rx} 2$ are the same as those used by $\mathrm{Tx} 1$ and $\mathrm{Tx} 2$, respectively, but the proposed scheme is implemented only in Rx1. The receiver Rx 3 only receives the output signal of $\mathrm{Tx} 1$, which is used as a reference. Besides, the receiver $\mathrm{Rx} 4$ receives the same signal as $\mathrm{Rx} 1$, but $\mathrm{Rx} 4$ uses the $\mathrm{LD}$ method for cancellation.

Fig. 3 shows that, in the beginning, the output signals of the DLL filter appear to fluctuate dramatically for a short period. It is because the receivers have not locked the signal from the transmitters at that time. Since $\mathrm{Rx} 1, \mathrm{Rx} 3$, and $\mathrm{Rx} 4$ are used to track the signal of $\mathrm{Tx} 1$, their output signals of the DLL filter during the signal locking process are the same, which are significantly different from $\mathrm{Rx} 2$.

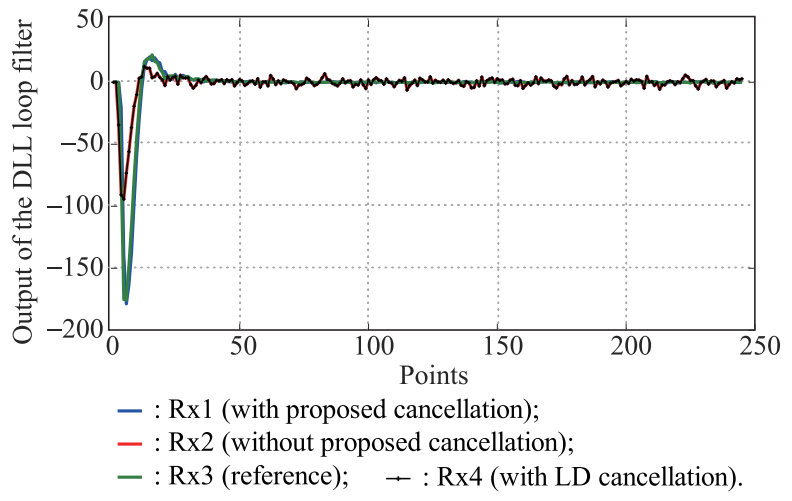

Fig. 3 Output signals of the DLL filter

Fig. 4 shows the details of Fig. 3. The jitters in Rx2 and $\mathrm{Rx} 4$ are significant, followed by that in $\mathrm{Rx} 1$. As mentioned above, since the LD method does not eliminate the MAI in DLL, the jitter in Rx4 is significant. Since no MAI exists in the received signal of Rx3, the jitter in Rx3 is the least. These simulation results are all in accordance with expectations.

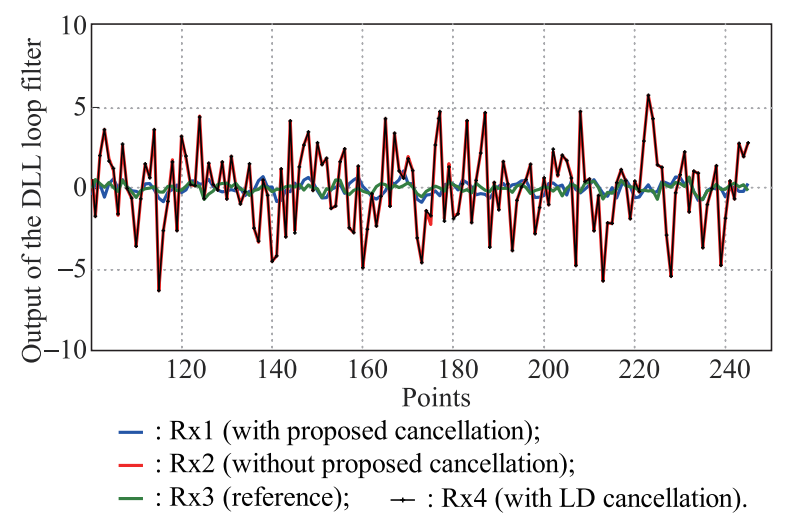

Fig. 4 Details of the DLL filter output

In the micro-satellite ranging system, the non-coherent pseudo-code ranging method is used often. Thus, it can be inferred that the relationship between the output of the DLL filter $\omega(t)$ and the distance $d$ is

$$
d=\frac{c}{2 f_{\mathrm{PN}}} \cdot \int_{0}^{t} \omega(t) T_{s} \mathrm{~d} t
$$

where $c$ is the speed of light propagation in the vacuum.

In Fig. 5, it can be seen that since the LD method does not eliminate the MAI in DLL, its standard deviation of the steady-state ranging error is almost identical to that without cancellation. It can also be seen that as SIR increases, the theoretical and simulated standard deviation of steadystate ranging error with and without the proposed scheme are both gradually decreased. Moreover, the ranging performance with cancellation is significantly improved compared to that without cancellation, especially at low SIRs. 
It can be seen that in the low input SIR region, the simulation curves deviate considerably from the theoretical ones. This is because when evaluating the theoretical ranging precision, only the carrier phase error residual is taken into account. At a low SIR, however, the carrier phase error residual may not be the dominating factor anymore.

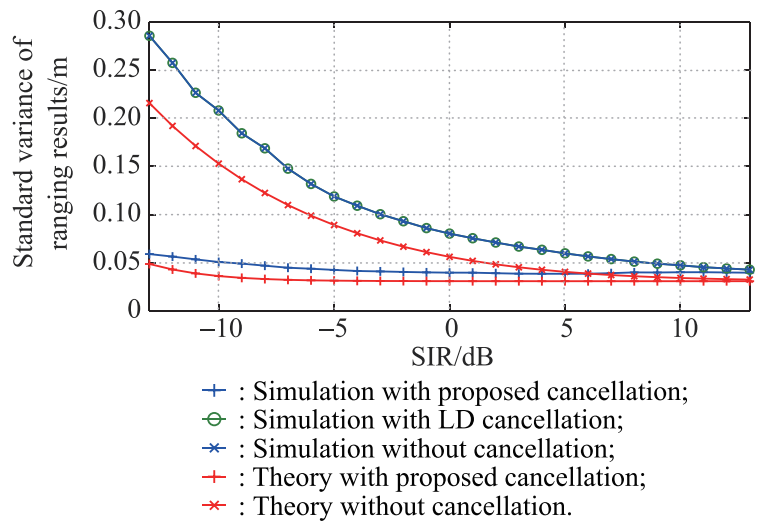

Fig. 5 Comparison between simulation and theory

\section{System demonstration}

In order to verify the previous analytical and simulation results, three digital baseband boards of transponders are used as the experimental platform. The main parameters of the experimental platform are shown in Table 2 where $P_{T}$ is the transmitting power of the transponder.

Table 2 Primary parameters of the experiment

\begin{tabular}{cc||cc}
\hline Parameter & Value & Parameter & Value \\
\hline$f_{s} / \mathrm{MHz}$ & 40 & $B_{L} / \mathrm{kHz}$ & 1 \\
$f_{c} / \mathrm{MHz}$ & 10 & $\xi_{L}$ & 0.7071 \\
$f_{\mathrm{PN}} / \mathrm{MHz}$ & 5.115 & $B_{L p} / \mathrm{Hz}$ & 200 \\
$f_{b} / \mathrm{kHz}$ & 5 & $\xi_{L p}$ & 0.7071 \\
$N$ & 1023 & $B_{f e} / \mathrm{MHz}$ & 20 \\
$P_{T} / \mathrm{dBm}$ & 20 & & \\
\hline
\end{tabular}

Two digital baseband broads are used for the slave satellites $\mathrm{A}$ and $\mathrm{B}$, and the third one is used for the master satellite in the cluster. The experimental platform is shown in Fig. 6.

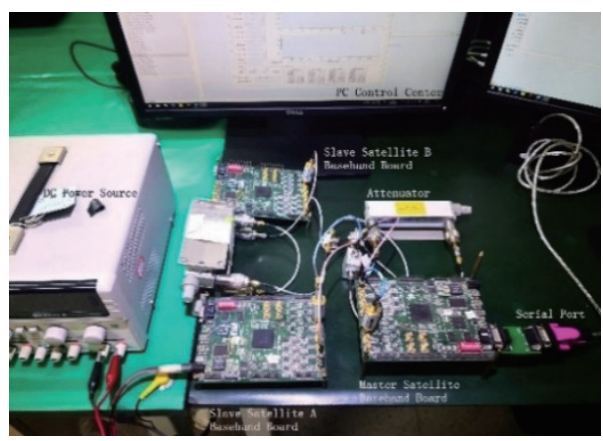

Fig. 6 Experimental platform
The coaxial cable is used to connect the master and slave satellites to avoid interference between the uplink and downlink. An adjustable attenuator at the transmitter port of satellite $A$ is used to adjust the output power, while the output power of the satellite B transmitter remains fixed. The acquisition of the measurement data and command control are implemented through a serial port between the master satellite and a computer.

The ranging results are shown in Fig. 7 and Fig. 8 for the input $\mathrm{SIR}=5 \mathrm{~dB}$ and $\mathrm{SIR}=-5 \mathrm{~dB}$, respectively.

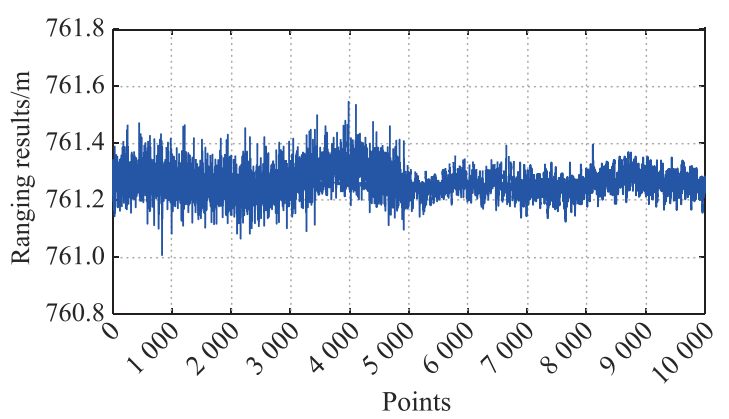

Fig. 7 Ranging results (SIR $=5 \mathrm{~dB})$

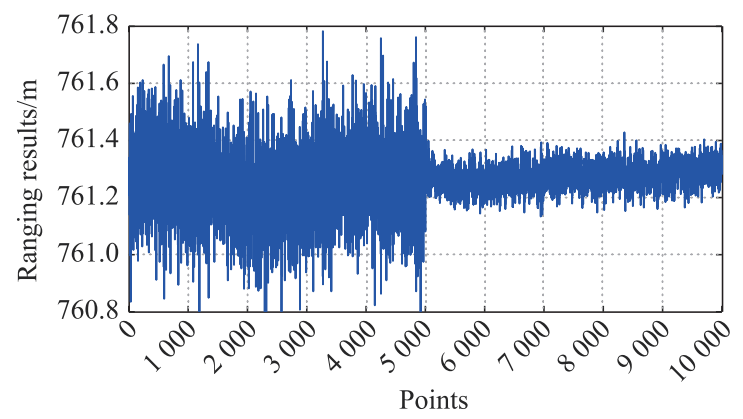

Fig. 8 Ranging results $(\mathrm{SIR}=-5 \mathrm{~dB})$

In order to compare the ranging performance between with and without the proposed scheme, when the process of data measurement is running to the middle, the proposed scheme is started to be executed. It can be seen that with the proposed scheme, the standard variance of the steadystate ranging error is reduced, as shown in Fig. 7 and Fig. 8. Moreover, the lower the input SIR, the more cancellation gain can be obtained. Under the conditions of SIR $=5 \mathrm{~dB}$ and SIR $=-5 \mathrm{~dB}$, the standard deviation of the steady-state ranging results decreases from $5.59 \mathrm{~cm}$ to $3.97 \mathrm{~cm}$ and $13.5 \mathrm{~cm}$ to $5.77 \mathrm{~cm}$, respectively. The results also prove that the proposed scheme can effectively improve the ranging precision.

Fig. 9 compares the theoretical and experimental standard variance of steady-state ranging results for different input SIR. It can be seen that in the low input SIR region, the experimental curves deviate significantly from the theoretical ones. The specific reason is the same as mentioned in the simulation result section. 


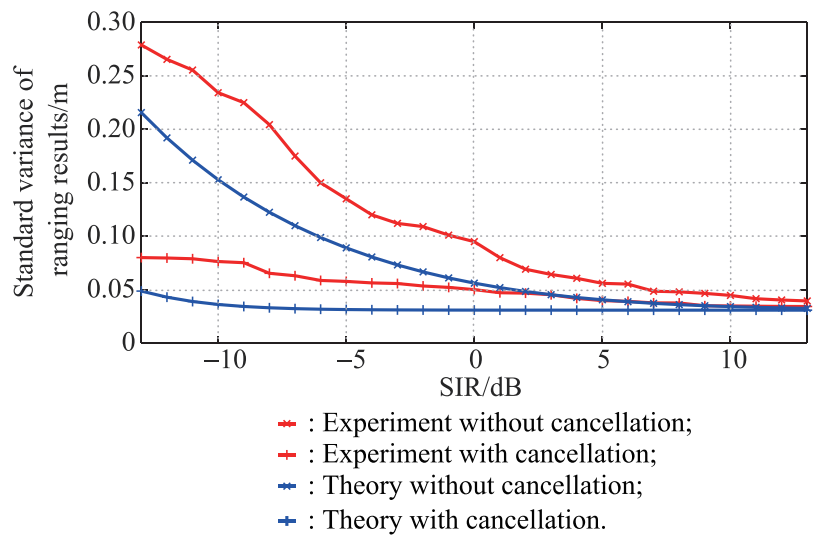

Fig. 9 Comparison between the experimental and theoretical results

\section{Conclusions}

In this paper, a parallel scheme for micro-satellite clusters based on the spread spectrum communication system is proposed. The scheme utilizes the information obtained from the interference channel to reconstruct the interference signal and then cancels it from the original delayed signal. Therefore, the SIR of the desired channel can be significantly improved compared to the LD. More importantly, the proposed scheme does not introduce considerable delays in the decision process compared to NLD, which guarantees the real-time performance of the system. The experimental results show that the standard deviation of the steady ranging error is decreased from $5.59 \mathrm{~cm}$ to $3.97 \mathrm{~cm}$ and $13.53 \mathrm{~cm}$ to $5.77 \mathrm{~cm}$, for the SIR = $5 \mathrm{~dB}$ and SIR $=-5 \mathrm{~dB}$ respectively.

\section{References}

[1] YIN J, CAO Y, LI Y H, et al. Satellite-based entanglement distribution over 1200 kilometers. Science, 2017, 356(6343): $1140-1144$.

[2] GALATIS G, GUO J, BUURSINK J. Development of a solar array drive mechanism for micro-satellite platforms. Acta Astronautica, 2017, 139: 407-418.

[3] MAHESHWARAPPA M R, BOWYER M D J, BRIDGES C P. Improvements in CPU and FPGA performance for small satellite SDR applications. IEEE Trans. on Aerospace and Electronic Systems, 2017, 53(1): 310-322.

[4] WANG Y, YE W, HONG Y, et al. Feasibility study: highly integrated chipset design for compact synthetic aperture radar payload on micro-satellite. Journal of Electromagnetic Waves and Applications, 2017, 31(6): 594-603.

[5] JIA M, LIU X, YIN Z, et al. Joint cooperative spectrum sensing and spectrum opportunity for satellite cluster communication networks. Ad Hoc Networks, 2017, 58(C): 231-238.

[6] YU Q Y, MENG W X, YANG M C, et al. Virtual multibeamforming for distributed satellite clusters in space information networks. IEEE Wireless Communications, 2016, 23(1): $95-101$.

[7] MIKHAILOV V N, KHACHATURIAN A B, MIKHEEV A S. CDMA signatures based on the shortened minimax binary sequences. Proc. of the Young Researchers in Electrical \& Electronic Engineering, 2017: $704-705$.
[8] YANG D, YANG J, LI G, et al. Globalization highlight: orbit determination using BeiDou inter-satellite ranging measurements. GPS Solutions, 2017, 21(3): 1395-1404.

[9] NAKASUKA S, MIYATA K, TSURUDA Y, et al. Discussions on attitude determination and control system for micro/nano/pico-satellites considering survivability based on Hodoyoshi-3 and 4 experiences. Acta Astronautica, 2018, 145: $515-527$.

[10] CHEGINI M, SADATI H, SALARIEH H. Chaos analysis in attitude dynamics of a flexible satellite. Nonlinear Dynamics, 2018, 93(3): $1421-1438$.

[11] PINTO F, AFGHAH F, RADHAKRISHNAN R, et al. Software defined radio implementation of DS-CDMA in intersatellite communications for small satellites. Proc. of the IEEE International Conference on Wireless for Space \& Extreme Environments, 2015: 1-6.

[12] GAYVORONSKIY D V, DANILCHUK E A. Analysis of multiple-access interference for CDMA signals. Proc. of the Young Researchers in Electrical \& Electronic Engineering, 2017: $144-145$.

[13] PUROHIT L S, AGARWAL A, THARANI L. An approach to compare the developments of MAI cancellation in multiuser detection of DS-CDMA system. Proc. of the International Conference on Soft Computing Techniques and Implementations, 2015: $81-84$.

[14] MOSHAVI S. Multi-user detection for DS-CDMA communications. IEEE Communications Magazine, 1996, 34(10): $124-136$.

[15] MANNA T, KOLE A. Performance analysis of secure DSSS multiuser detection under near-far environment. Proc. of the International Conference on Intelligent Control Power and Instrumentation, 2016: 258-262.

[16] VARANASI M K, AAZHANG B. Near-optimum detection in synchronous code-division multiple-access systems. IEEE Trans. on Communications, 1991, 39(5): 725 -736.

[17] VOICU C, HALUNGA S, VIZIREANU N. Performances of decorrelating MUD using STBC for image transmissions in rayleigh fading channel. Proc. of the International Conference on Telecommunication in Modern Satellite, 2013, 2: $597-$ 600.

[18] LUPAS R, VERDU S. Linear multiuser detectors for synchronous code-division multiple-access channels. IEEE Trans. on Information Theory, 1989, 35(1): $123-136$.

[19] WU Z L, YIN L, CUI K, et al. A FPGA implementation of MMSE MUD algorithm for TDRSS. Proc. of the 4th International Conference on Instrumentation \& Measurement, 2014: $427-430$.

[20] KAUR T, ARORA M. A dynamic successive interference cancellation (DSIC) scheme for latency reduction in MC-CDMA multiuser detection. Proc. of the International Conference on Next Generation Computing Technologies, 2016: 414-419.

[21] DIVSALAR D, SIMON M K, RAPHAELI D. Improved parallel interference cancellation for CDMA. IEEE Trans. on Communications, 1998, 46(2): 258-268.

[22] BETZ J W, KOLODZIEJSKI K R. Generalized theory of code tracking with an early-late discriminator part II: noncoherent processing and numerical results. IEEE Trans. on Aerospace and Electronic Systems, 2009, 45(4): $1557-1564$.

[23] RYCROFT M J. Understanding GPS. Principles and applications. Journal of Atmospheric and Solar-Terrestrial Physics, 1997, 59(5): $598-599$.

[24] PARKINSON B W. Global positioning system: theory and applications, Volume II. USA: AIAA, 1996.

[25] LEE Y H, KIM S J. Sequence acquisition of DS-CDMA systems employing gold sequences. IEEE Trans. on Vehicular 
Technology, 2000, 49(6): 2397-2404.

[26] GONG M, LIN T. Research of narrow-band interference suppression technologies in DSSS communication systems. Proc. of the International Conference on Wireless Communications, 2009: $1-5$.

[27] MOSTAFA HJING G. Simulink implementation of a CDMA MIMO-beamforming system. Proc. of the International Symposium on Antenna Technology \& Applied Electromagnetics, 2005: $1-4$.

\section{Biographies}

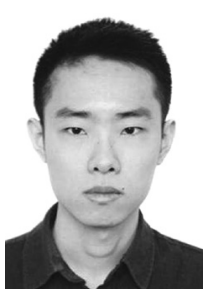

HUANG Jiajun was born in 1994. He received his B.E. degree from Dalian Maritime University. He is a Ph.D. candidate of the Micro-Satellite Research Center, Zhejiang University. His research interests include micro-transponder, inter-satellite ranging, and inband full-duplex.

E-mail: 11624025@zju.edu.cn

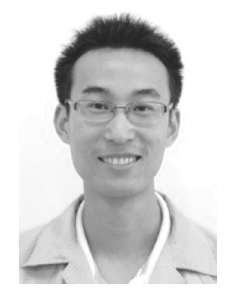

ZHANG Chaojie was born in 1982. He received his B.E. and Ph.D. degrees from Zhejiang University in 2004 and 2009, respectively. Now he is an associate researcher at Zhejiang University. He has joined in the Micro-Satellite Research Center since he was a graduate student. His research interests include micro-satellite and software-defined radio technologies.

E-mail: zhangcj@zju.edu.cn

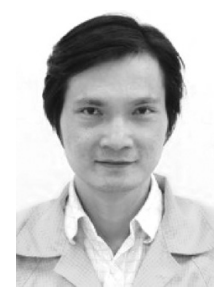

JIN Xiaojun was born in 1977. He received his B.E, M.E. and Ph.D. degrees from Zhejiang University in 2001, 2004 and 2007, respectively. He joined the faculty of Zhejiang University in 2009 and has been an associate professor since 2010. His research interests include micro-satellite transponder technology, relative ranging, and navigation of micro-satellite formation flying.

E-mail: axemaster@zju.edu.cn 